\title{
Results of a Minimally Invasive Technique for Treatment of Unicameral Bone Cysts
}

\author{
Gökçe Mik MD, Alexandre Arkader MD, \\ Alexander Manteghi BS, John P. Dormans MD
}

Received: 1 October 2008/Accepted: 17 July 2009/Published online: 4 August 2009

(C) The Association of Bone and Joint Surgeons (B) 2009

\begin{abstract}
Unicameral bone cysts are benign bone lesions commonly seen in pediatric patients. Several treatment methods have been described with variable results and high recurrence rates. We previously reported short-term success of a minimally invasive technique that includes combining percutaneous decompression and grafting with medical-grade calcium sulfate pellets. The purpose of this
\end{abstract}

Each author certifies that he or she has no commercial associations (eg, consultancies, stock ownership, equity interest, patent/licensing arrangements, etc) that might pose a conflict of interest in connection with the submitted article.

Each author certifies that his or her institution has approved the human protocol for this investigation, that all investigations were conducted in conformity with ethical principles of research, and that informed consent for participation in the study was obtained.

This work was performed at The Children's Hospital of Philadelphia.

\section{G. Mik}

Department of Orthopaedic Surgery, Istanbul Cerrahi

Hastanesi, Istanbul, Turkey

e-mail: gokcemik@hotmail.com

\author{
A. Manteghi \\ Department of Orthopaedics, The Children's Hospital \\ of Philadelphia, Philadelphia, PA, USA
}

\author{
A. Arkader \\ Children's Orthopaedic Center, Children's Hospital \\ Los Angeles, and Keck School of Medicine, University \\ of Southern California, Los Angeles, CA, USA
}

\section{J. P. Dormans}

Department of Orthopaedic Surgery, University of Pennsylvania

School of Medicine, Philadelphia, PA, USA

\section{J. P. Dormans $(\bowtie)$}

Department of Orthopaedic Surgery, The Children's Hospital of Philadelphia, 2nd Floor Wood Center, 34th and Civic Center Blvd, Philadelphia, PA 19104-4399, USA

e-mail: dormans@email.CHOP.edu study was to review the additional long-term results with a minimum followup of 24 months (average, 37 months; range, 24-70 months). We identified 55 patients with an average age of 10.8 years (range, 1.3-18 years). Forty-one of 55 lesions occurred in the humerus and femur. Fortyfour of $55(80 \%)$ patients had a partial or complete response after initial surgery; of these, seven obtained a partial or complete response after a repeat surgery (cumulative healing rate, 94\%). Two patients underwent a third surgery (cumulative healing rate, 98\%). One underwent a third repeat surgery (cumulative healing rate, $100 \%)$. There were no major complications associated with the procedure. Two patients had a superficial infection that resolved with oral antibiotics. Although some patients required a repeat procedure, complete or partial response at a minimum 24 months' followup was achieved in all patients.

Level of Evidence: Level IV, therapeutic study. See Guidelines for Authors for a complete description of levels of evidence.

\section{Introduction}

Unicameral bone cysts (UBCs) are among one of the most common benign bone lesions in pediatric patients, representing approximately $3 \%$ of all bone tumors [2]. Although several treatments have been described, the intermediateto long-term results are variable and many of the patients (20\%-70\%) need at least a second intervention owing to recurrence or persistence $[8,16,17,19,22,25]$. A new minimally invasive technique for UBCs that combines percutaneous decompression and grafting with medicalgrade calcium sulfate (MGCS) pellets was described previously, with very encouraging short-term results [11]. 
The purpose of the current study was to assess the long-term followup based on the previous study, expanding the cohort, to validate the efficacy of the described technique. We specifically sought to determine the incidence of healing of UBCs and to assess the resolution of pain and return of function for these patients. Finally, we wished to detect any relationship between the risk of cyst persistence/ recurrence and the need for multiple procedures in relation to the age of the patients, presence of pathologic fracture, and cyst activity as assessed by the Neer criteria [18].

\section{Materials and Methods}

After obtaining approval from the Institutional Review Board, the prospective musculoskeletal tumor database from a tertiary pediatric center was queried to identify all children and adolescents diagnosed with a UBC between January 2000 and June 2005 . We included only patients who were treated by a minimally invasive technique as described by
Dormans et al. $[10,11]$. We identified 55 children who underwent this minimally invasive technique for UBCs. There were 36 boys and 19 girls, with an average age at the time of surgery of 10.8 years (range, $1.3-18$ years). The minimum followup for inclusion was 24 months.

The diagnosis of UBC initially was made based on characteristic radiographic features (Fig. 1A-B). Thirty-two of 55 patients (58\%) presented with a pathologic macrofracture (ie, visible on radiographs), 18 of 55 patients $(33 \%)$ presented with local pain (presumed microfracture, not visible on radiographs), and in five of 55 patients (9\%), the cyst was diagnosed incidentally. On plain radiographs, the UBC was seen as a well-defined, lytic, central lesion, with no periosteal reaction or other signs of aggressiveness. Cortical thinning almost always was seen with long-bone lesions and pathologic macrofracture was present in 33 of 55 patients (60\%). The humerus was involved in 29 of 55 patients $(53 \%)$, including 15 proximal-third cysts, 13 middle-third cysts, and one distal-third cyst. The femur was involved in 12 of 55 patients (22\%), including seven proximal-third cysts, four
Fig. 1A-G A 13-year-old boy had a persistent UBC of the humerus after two steroid injections at an outside institution. (A) A preoperative anteroposterior radiograph shows the UBC. (B) A followup image taken 6 months after a first minimally invasive procedure shows the patient had an incomplete response, requiring a second surgery. (C) Intraoperative fluoroscopy shows the cystogram confirming the residual chamber; (D) a pituitary rounger was used to remove the cyst lining; the intramedullary decompression was performed using an angled curette (E) proximally (F) and distally. (G) The final result is seen after insertion of the MGCS pellet. The pellets extend beyond the cyst wall, confirming full decompression of the cavity.
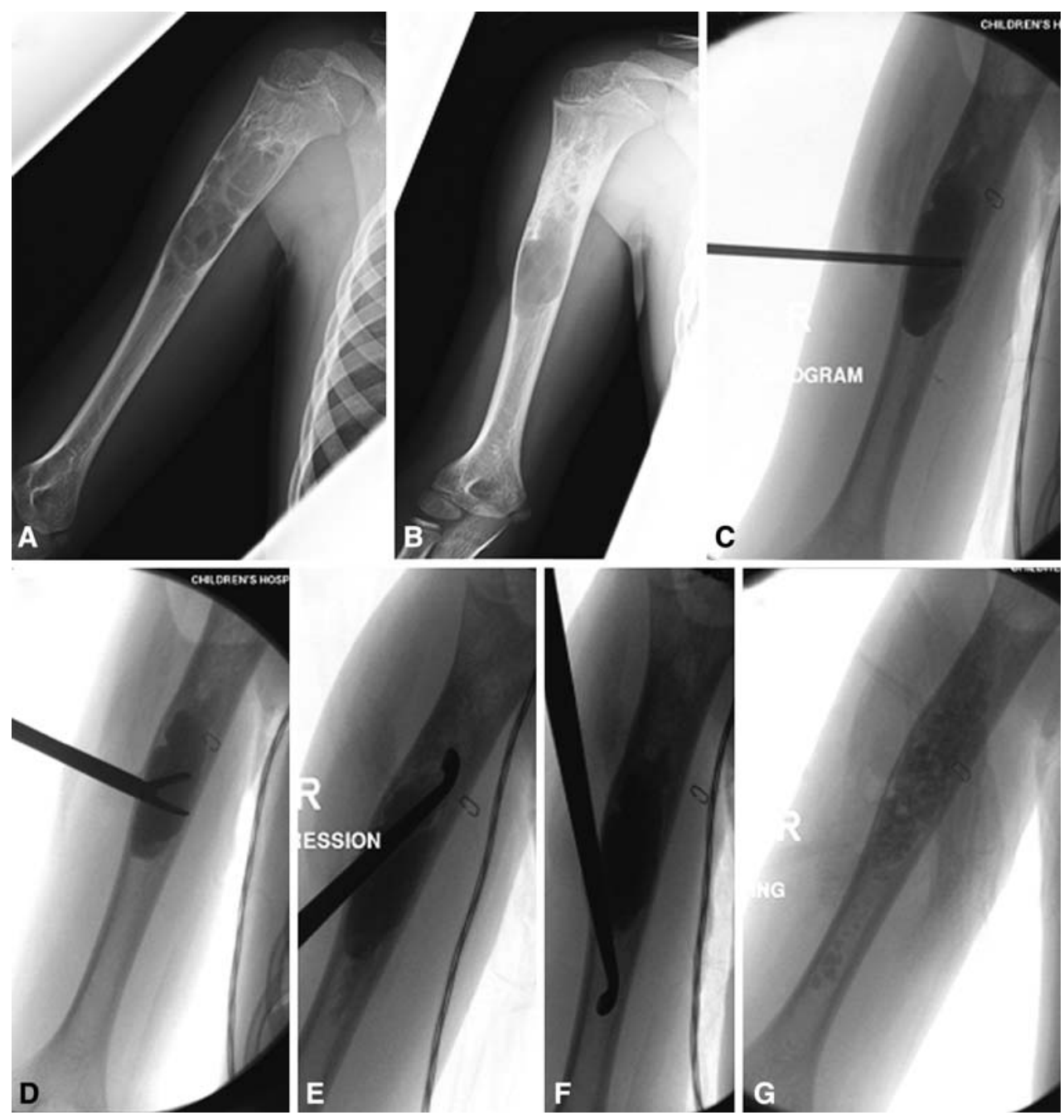
Table 1. Anatomic distribution of the lesions

\begin{tabular}{lc}
\hline Location of the cyst & Number of lesions \\
\hline Humerus & 29 \\
$\quad$ Proximal third & 15 \\
$\quad$ Middle third & 1 \\
$\quad$ Distal third & 13 \\
Femur & 12 \\
$\quad$ Proximal third & 7 \\
$\quad$ Middle third & 4 \\
$\quad$ Distal third & 1 \\
Calcaneus & 9 \\
Fibula & 2 \\
Tibia & 1 \\
Clavicle & 1 \\
Radius & 1 \\
Total & 55 \\
\hline
\end{tabular}

middle-third cysts, and one distal-third cyst. The calcaneus was involved in nine of 55 patients (16\%); two of 55 patients (3.5\%) had fibula cysts; and the tibia, radius, and clavicle each were involved in one patient (Table 1).

Of the 55 patients, 11 had been treated previously elsewhere and presented with a recurrent/persistent lesion. Of these, four had undergone curettage and allografting, six had had multiple steroid injections, and one had received Grafton ${ }^{\circledR}$ (Osteotech, Eatontown, NJ) injection.

Intraoperatively, the diagnosis was confirmed or supported by (1) aspiration of clear fluid (serous or seroussanguineous) followed by (2) a cystogram (identification of one chamber containing water-soluble radiopaque material). Whenever the diagnosis was uncertain, incisional biopsy of the cyst lining and intraoperative frozen section were performed.

The surgical indications included symptomatic lesions, painful or large recurrent tumors treated elsewhere, and large lesions associated with substantial cortical thinning and high risk of pathologic fracture.

The procedure was performed with the patient under general anesthesia. After localization of the lesion by fluoroscopy, a sharp Jamshidi trocar needle (Cardinal Health, Dublin, $\mathrm{OH}$ ) was introduced percutaneously into the middle of the cyst cavity, preferably through the thinnest area of the cyst wall. The cyst was aspirated and a cystogram using water-soluble dye was performed to observe the single chamber, characteristic for UBC (Fig. 1C). After diagnostic confirmation, a 0.5 - to 1 -cm longitudinal incision was made over the aspiration site. An entry portal through the cyst wall was created using a 6-mm sharp arthroscopy trocar to allow passage for curettes. The cyst lining was removed with a large pituitary rounger (Fig. 1D) and different-sized angled curettes under fluoroscopy. A sample tissue was sent for frozen

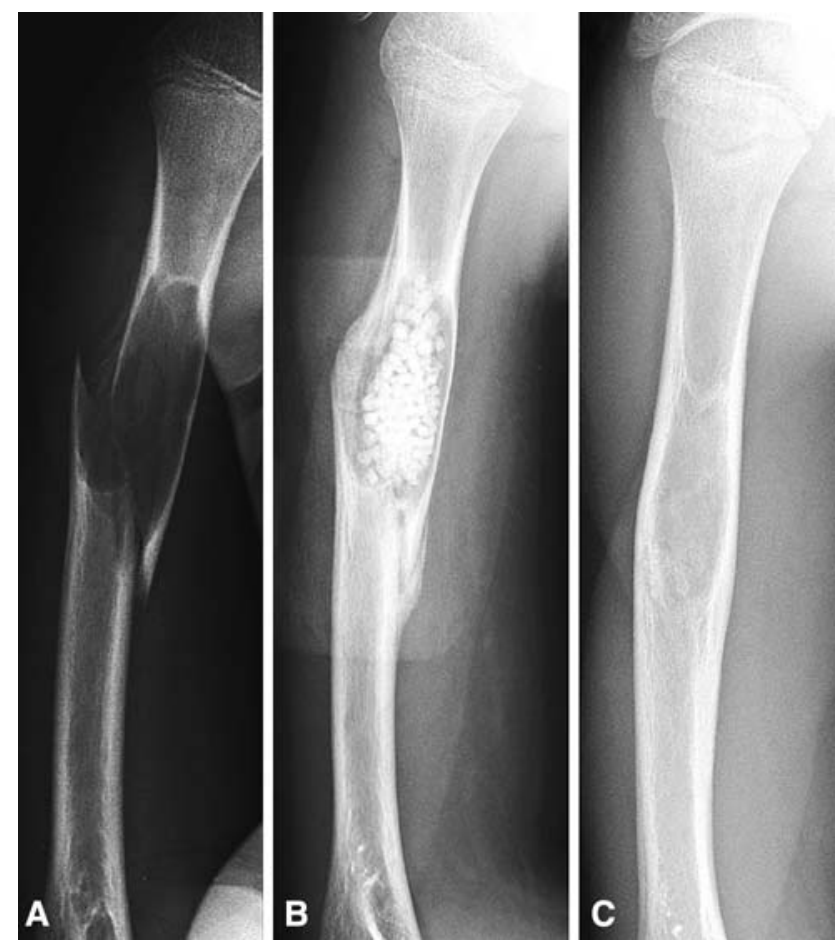

Fig. 2A-C A 12-year-old boy sustained a pathologic fracture through a UBC of the proximal humerus. (A) His initial radiograph shows a displaced fracture treated nonoperatively. (B) After fracture healing (6 weeks), the patient underwent a minimally invasive procedure. (C) Twelve weeks after surgery, the graft has been completely reabsorbed, there is a complete response to the surgery, and the humerus already is remodeling.

section when there was question about the diagnosis. Intramedullary decompression was performed using a flexible titanium nail or an angled curette (Fig. 1E-F). Continuity of the cyst cavity with the normal bone marrow was confirmed with an image intensifier. The cavity then was packed with MGCS pellets (Osteoset ${ }^{\circledR}$; Wright Medical Technology, Arlington, TN). The pellets are radiopaque and complete filling of the cavity can be monitored (Fig. 1G). The incision was closed in layers. In the presence of a preoperative pathologic fracture, immobilization was applied for 3 to 6 weeks before surgical intervention (Fig. 2).

The medical charts were reviewed retrospectively for demographic information, including gender and age; clinical presentation; diagnostic modalities, including all available imaging examinations; operative records; complications; and clinical and radiographic outcomes, including the presence of residual symptoms, range of motion, muscle strength, and return to activities at last followup. The senior author (JPD) was not involved in the radiographic or data analysis to avoid any potential bias, related to his role in the development of the technique.

The preoperative radiographs were reviewed and the cysts were classified according to the criteria of Neer et al. 
Table 2. Radiographic response to surgical treatment [11]

\begin{tabular}{ll}
\hline Response & Description \\
\hline Complete healing & $\begin{array}{l}\text { Opacification of the cyst }>95 \%+\text { cortical } \\
\text { thickening } \\
\text { Opacification of the cyst }>80 \% \pm \text { cortical } \\
\text { thickening }\end{array}$ \\
Partial healing & $\begin{array}{l}\text { Opacification of the cyst }<80 \% \\
\text { No changes } \\
\text { No response }\end{array}$ \\
Yes/no & Need for additional surgery
\end{tabular}

(Adapted and published with permission from Lippincott Williams \& Wilkins from: Dormans JP, Sankar WN, Moroz L, Erol B. Percutaneous intramedullary decompression, curettage, and grafting with medical-grade calcium sulfate pellets for unicameral bone cysts in children: a new minimally invasive technique. J Pediatr Orthop. 2005;25:804-811.)

[18]. In brief, an active cyst was defined as one in which the margin of the cyst was in close proximity to the epiphyseal plate, and a latent cyst was separated from the growth plate by a segment of normal bone. Postoperatively, the radiographs were evaluated using the classification system described by Dormans et al. [11]. Also, the need for additional surgery based on the radiographic result, location of the lesion, and clinical symptoms was collected and annotated (Table 2).

We evaluated several factors, including demographics, clinical presentation (presence of pathologic fracture), and cyst stage and location to determine whether there were any identifiable prognosticators of persistence or incomplete response after the minimally invasive surgical approach.

Statistical analysis included descriptive analysis and a two-sample $t$ test to determine whether there was a significant difference between patients needing repeat surgery and patients not needing repeat surgery.

\section{Results}

At final followup, all patients were considered healed, with no evidence of clinically important recurrence or persistence of their cyst. Fifty-five patients underwent a total of 74 minimally invasive procedures. After the initial procedure, 44 of 55 patients $(80 \%$ ) had complete ( 38 of 55, 69\%) or partial (six of 55,11\%) healing of their cyst and did not warrant further treatment. If only lesions of long bones were included, the complete healing rate was $78 \%$ (36 of $55)$. Eleven of 55 (20\%) patients underwent repeat surgery. After the second surgery, seven of 11 patients had complete healing of their cyst and another had partial healing. The cumulative healing rate after the second procedure was 94\% (52 of 55). Two patients achieved complete response after a second repeat surgery, for a cumulative healing rate of $98 \%$ (54 of 55). One patient needed a third repeat surgery to achieve complete healing. The final cumulative healing rate was $100 \%$ for the entire cohort.

Clinically, all patients recovered preoperative range of motion and muscle strength, fully returning to their daily activities within 6 to 12 weeks after the surgery. No patients had residual pain or tenderness at the UBC site. There were no major complications associated with the procedure. Two of 55 patients (two of 79 procedures, $2.5 \%$ ) had superficial drainage that rapidly resolved with oral cephalexin. The superficial infection was a clinical diagnosis and no cultures were performed. There were no deep or persistent infections. There were no known adverse reactions/allergies to the MGCS pellets, although it is unclear if the infections were actually a reaction to the pellets, represented by drainage. Three patients sustained a pathologic fracture (two macrofractures and one microfracture) between initial surgery and a repeat procedure. Two of them experienced a high-energy trauma (motorcycle and go-cart accident), and the third patient had a low-energy trauma that could represent transitory bone weakness after the surgery (cortical window).

We evaluated several of the possible prognostic factors for persistence and/or incomplete response but found no factors that could predict recurrence or need for multiple procedures. Variables such as patient age, presence of a pathologic fracture, and Neer classification of cyst activity were not predictive of the need for additional surgery. The average age of the patients who had complete or partial healing after the first surgical procedure was 11.3 years (range, 2.8-18 years), whereas the average age of the patients requiring repeat surgery for incomplete response or no response was 8.7 years (range, 4.5-16 years) $(p=0.055)$. There was a relatively higher $(p>0.1)$ incidence of pathologic fractures among the patients needing a second surgery compared with patients who achieved healing after the initial procedure, 77\% (10 of 13) versus $52 \%$ (22 of 42), respectively. According to the Neer classification, 33 of 46 cysts (72\%) in the long bones were latent and 13 of $46(28 \%)$ were active. Of those, eight of 33 latent cysts (18\%) and five of 13 (29\%) active cysts required repeat surgery $(\mathrm{p}=0.41)$. Cyst location also did not influence the persistence rate.

\section{Discussion}

In view of the fact that there is no standardized treatment for UBCs, we performed this retrospective study to assess the long-term followup of our minimally invasive technique that combines percutaneous cyst decompression, curettage, and grafting using MGCS pellets. Different centers and surgeons have different approaches, with variable rates of 
cyst resolution and recurrence/persistence $[1,3,4,6,8,9$, $11,14,16,17,20,22,23]$. It generally is accepted that radical excision of the cyst reduces the rate of recurrence but increases the morbidity and complication rate $[8,15$, 18]. As UBCs are benign bone lesions that do not necessarily warrant surgical intervention, such an invasive approach is not recommended. In our preliminary study, we reported a $95 \%$ short-term success rate with the minimally invasive technique (eg, complete or partial healing/ opacification) [11]. However, when we reviewed the intermediate- to long-term results in a larger cohort, the observed rate of complete or partial response achieved with one surgical intervention was $80 \%$. Nevertheless, the success rate increased to $94 \%$ after a repeat surgery, reaching a $100 \%$ healing rate in patients undergoing more than two repeat surgeries. These intermediate- to long-term results reflect the limitation of achieving a $100 \%$ healing rate for UBCs after only one minimally invasive procedure $[3,4,14,22]$.

Our study has several limitations. Although we could not find any prognostic factor for cyst persistence/recurrence, the patients requiring additional surgery tended to be younger, have a higher incidence of pathologic fracture at presentation, and have a higher rate of active cysts. It is unclear regarding whether a larger cohort would provide appropriate statistical power to identify any of these factors as truly significant. Other limitations of our study include its retrospective nature and the absence of a direct comparative group or randomized comparison. Also, some patients had a shorter radiographic than clinical followup and there was no blinded review of the films.

Scaglietti [21] initially described the use of steroid injection for UBCs in 1974; 8 years later, Scaglietti et al. reported long-term results with this technique [22]. Complete cyst obliteration was observed in 55\% (83 of 163) of the cases; $76 \%$ of the patients needed at least a second procedure [22]. Others also have reported on the Scaglietti technique, with variable success rates $(32 \%-80 \%)$ [3, 14, 22].

Numerous authors have tried injecting different graft materials into the cyst cavity [9, 13, 16, 17, 20, 25]. Autologous bone marrow injection has been used, with healing rates varying from $50 \%$ to $75 \%$ [4, 6, 9, 25]. In a study of 23 patients undergoing cyst trephination and injection of allogeneic demineralized bone matrix and autologous bone marrow, after an average 50 months' followup, five $(22 \%)$ required a second procedure and seven $(30 \%)$ had incomplete healing [20]. Although the concept of using MGCS pellets was described previously [24], the combination of intramedullary decompression followed by MCGS grafting initially was proposed by the senior author (JPD). The MCGS is a fairly inexpensive bone substitute that is osteoconductive and helps fill the defect and monitor response.
Although the etiology of UBCs is still unknown, among the accepted theories is the concept described by Cohen [7] in 1960 that UBCs have an elevated hydrostatic pressure and therefore a decompression of the cavity is essential to obtain healing $[5,7]$. We believe intramedullary decompression is the key to success (decreases intracyst pressure and provides bone marrow grafting). Others also have highlighted the significance of opening the medullary canal in the surgical treatment of UBCs [1, 12]. Several methods of continued cyst decompression have been described including the use of Kirschner wires, cannulated screws, and intramedullary titanium elastic nails to provide continuous decompression and stability [1, 8, 12, 19]. In a review of 32 patients treated by the flexible intramedullary nailing technique, $94 \%$ of the patients reportedly achieved healing, but change of nails was required in $28 \%$ and extraction of the nails was performed in all [19]. In another study of the use of the same technique in 47 patients, $66 \%$ (31 of 47) achieved complete healing, 30\% (14 of 47) required a second surgery for removal of the nails, and two had growth disturbance [8]. We do not believe additional stability (internal fixation) is needed after the minimally invasive technique. Exceptions are lesions located in the proximal femur, those involving the majority of the metaphyseal diameter, and those that are unstable and associated with macrofracture. Patients presenting with a pathologic fracture are treated nonoperatively for approximately 3 to 6 weeks and then undergo the minimally invasive technique.

Based on these results, we believe it is possible to obtain adequate healing using a minimally invasive technique for treatment of UBCs. A larger cohort in a randomized prospective study is needed to compare these results directly with results of other techniques and prove its efficacy. The potential advantages of this technique include the percutaneous approach, short operative time, and short learning curve. MGCS pellets seem to be safe, are fairly inexpensive, are reabsorbed in a short time, and are radiopaque facilitating radiographic followup. Although the healing rate of patients with these intermediate- and longterm results was less than that in a preliminary report, percutaneous intramedullary decompression, curettage, and grafting with MGCS pellets may be an effective treatment for UBCs, achieving healing in most patients with no major complications.

\section{References}

1. Bumci I, Vlahovic T. Significance of opening the medullar canal in surgical treatment of simple bone cyst. J Pediatr Orthop. 2002;22:125-129.

2. Campanacci M, Capanna R, Picci P. Unicameral and aneurysmal bone cysts. Clin Orthop Relat Res. 1986;204:25-36. 
3. Capanna R, Dal Monte A, Gitelis S, Campanacci M. The natural history of unicameral bone cyst after steroid injection. Clin Orthop Relat Res. 1982;166:204-211.

4. Chang CH, Stanton RP, Glutting J. Unicameral bone cysts treated by injection of bone marrow or methylprednisolone. J Bone Joint Surg Br. 2002;84:407-412.

5. Chigira M, Maehara S, Arita S, Udagawa E. The aetiology and treatment of simple bone cysts. J Bone Joint Surg Br. 1983;65: 633-637.

6. Cho HS, Oh JH, Kim HS, Kang HG, Lee SH. Unicameral bone cysts: a comparison of injection of steroid and grafting with autologous bone marrow. J Bone Joint Surg Br. 2007; 89:222226.

7. Cohen J. Simple bone cysts: studies of cyst fluid in six cases with a theory of pathogenesis. J Bone Joint Surg Am. 1960;42:609-616.

8. de Sanctis N, Andreacchio A. Elastic stable intramedullary nailing is the best treatment of unicameral bone cysts of the long bones in children?: prospective long-term follow-up study. J Pediatr Orthop. 2006;26:520-525.

9. Docquier PL, Delloye C. Treatment of simple bone cysts with aspiration and a single bone marrow injection. J Pediatr Orthop. 2003;23:766-773.

10. Dormans JP, Dormans NJ. Use of percutaneous intramedullary decompression and medical-grade calcium sulfate pellets for treatment of unicameral bone cysts of the calcaneus in children. Orthopedics. 2004;27(1 suppl):s137-s139.

11. Dormans JP, Sankar WN, Moroz L, Erol B. Percutaneous intramedullary decompression, curettage, and grafting with medical-grade calcium sulfate pellets for unicameral bone cysts in children: a new minimally invasive technique. J Pediatr Orthop. 2005;25:804-811.

12. Givon U, Sher-Lurie N, Schindler A, Ganel A. Titanium elastic nail: a useful instrument for the treatment of simple bone cyst. J Pediatr Orthop. 2004;24:317-318.

13. Glaser DL, Dormans JP, Stanton RP, Davidson RS. Surgical management of calcaneal unicameral bone cysts. Clin Orthop Relat Res. 1999;360:231-237.
14. Hashemi-Nejad A, Cole WG. Incomplete healing of simple bone cysts after steroid injections. J Bone Joint Surg Br. 1997;79:727730 .

15. Herring JA, Peterson HA. Simple bone cyst with growth arrest. J Pediatr Orthop. 1987;7:231-235.

16. Inoue O, Ibaraki K, Shimabukuro H, Shingaki Y. Packing with high-porosity hydroxyapatite cubes alone for the treatment of simple bone cyst. Clin Orthop Relat Res. 1993;293:287-292.

17. Lokiec F, Ezra E, Khermosh O, Wientroub S. Simple bone cysts treated by percutaneous autologous marrow grafting: a preliminary report. J Bone Joint Surg Br. 1996;78:934-937.

18. Neer CS 2nd, Francis KC, Marcove RC, Terz J, Carbonara PN. Treatment of unicameral bone cyst: a follow-up study of one hundred seventy-five cases. J Bone Joint Surg Am. 1996;48: 731-745.

19. Roposch A, Saraph V, Linhart WE. Flexible intramedullary nailing for the treatment of unicameral bone cysts in long bones. J Bone Joint Surg Am. 2000;82:1447-1453.

20. Rougraff BT, Kling TJ. Treatment of active unicameral bone cysts with percutaneous injection of demineralized bone matrix and autogenous bone marrow. J Bone Joint Surg Am. 2002;84: 921-929.

21. Scaglietti O. Sull' azione osteogenice dell' acetato di prednisolone. Boll Soc Tosco-Umbra Chir. 1974;35:35-41.

22. Scaglietti O, Marchetti PG, Bartolozzi P. Final results obtained in the treatment of bone cysts with methylprednisolone acetate (depo-medrol) and a discussion of results achieved in other bone lesions. Clin Orthop Relat Res. 1982;165:33-42.

23. Spence KF Jr, Bright RW, Fitzgerald SP, Sell KW. Solitary unicameral bone cyst: treatment with freeze-dried crushed cortical-bone allograft. A review of one hundred and forty-four cases. J Bone Joint Surg Am. 1976;58:636-641.

24. Wilkins RM. Unicameral bone cysts. J Am Acad Orthop Surg. 2000;8:217-224.

25. Yandow SM, Lundeen GA, Scott SM, Coffin C. Autogenic bone marrow injections as a treatment for simple bone cyst. J Pediatr Orthop. 1998;18:616-620. 